\title{
Strong Law of Large Numbers for Weighted Sum of Exchangeable Random Variables
}

\author{
Zhaoxia Huang (Corresponding author) \\ Department of Mathematics, Ankang University, Ankang 725000, China \\ E-mail: huangdoudou3333@163.com
}

Special funds project of scientific research in Ankang University, No. AYQDZR200905

\begin{abstract}
In this paper, the Marcinkiewicz type theorem is extended to the case of exchangeable random variables. As a generalization, we also obtain two strong laws of large numbers on the weighted sum of exchangeable random variables
\end{abstract}

Keywords: Exchangeability, Law of large numbers

\section{Introduction}

If the replacement the joint distribution of $X_{1}, \ldots, X_{n}$ is unchanged, that is, for each replacement $\pi$ of $1,2, \ldots n$, the joint distribution of $X_{1}, \ldots, X_{n}$ is the same with that of $X_{\pi(1)}, \ldots, X_{\pi(n)}$; then the random variable finite series $X_{1}, \ldots$, $X_{n}$ is known as the exchangeable. Obviously, the independent identical distribution random variables is the simplest exchangeable random variables. The concept of exchangeable random variables is the first proposed by De Finetti 1930. The most famous property of exchangeable random variables is its basic structured theorem, called De Finetti theorem; that is, the infinite series of exchangeable random variables is independent identical distribution, if its tail is $\sigma$ algebra.

The aim of this paper is generalize the independent identical distribution variables Bai, 2000, P.105-112 and Sung, 2001, P.413-419 to the exchangeable random variables. As the selection method for truncated random variables is different when deal with random variables, so the prove method is more simple than that of Bai, 2000, P.105-112 and Sung, 2001, P.413-419.

Definition $\mathrm{Wu}, 2006, \mathrm{P} .132-133$. The positive valued function $l(x)$ defined on $[, \infty)$ is called slowly changed, if for any $c>0$, we have $\lim _{x \infty} \frac{l(c x)}{l(x)}$.

Suppose $\left\{a_{n i}, 1 \leq i \leq n, n \geq 1\right\}$ is real positive series that satisfy $A_{\alpha, n}^{\alpha} \frac{1}{n} \sum_{i=1}^{n}\left|a_{n i}\right|^{\alpha}$, and

$$
A_{\alpha}=\lim _{n \rightarrow \infty} \sup A_{\alpha}, \quad n<\infty .
$$

Lemma Wu, 2006, P.132-133. Suppose $\left\{X_{n}, n \geq 1\right\}$ are exchangeable random variables, that satisfy

$$
\operatorname{Cov}\left(f_{1}\left(X_{1}\right), f_{2}\left(X_{2}\right)\right) \leq 0 .
$$

Let $A_{1}, \ldots, A_{m}$ be the disjoint non-empty subset of $\{1,2, \ldots, n\}$, with $m \geq 2$. Suppose $f_{i}, i=1,2, \ldots m$ is a non-increase (non-decrease) function, then

(1). If $f_{i} \geq 0, i=1, \ldots, m$ then

$$
E \prod_{i=1}^{n} f\left(X_{j}, j \in A_{i}\right) \leq \prod_{i=1}^{n} E f\left(X_{j}, j \in A_{i}\right) .
$$

(2). Particularly, for any $x_{i} \in R, i=1, \ldots, m$, we have

$$
P\left(X_{1}<x_{1}, \ldots, X_{m}<x_{m}\right) \leq \prod_{i=1}^{n} P\left(X_{i}<x_{i}\right) .
$$

Subsequently, we will outline several lemmas, which will be used in the proof of the main theorems. If necessary, we will also give the proof. 
Lemma 2. Suppose $X_{1}, \ldots, X_{n}$ are exchangeable random variables, that satisfy

$$
\operatorname{Cov}\left(f_{1}\left(X_{1}\right), f_{2}\left(X_{2}\right)\right) \leq 0
$$

and $E X_{k}=0, \sigma_{k}^{2}=E X_{k}^{2}<\infty(k=1,2, \ldots, n)$. Suppose there exists a positive constant $H$ such that

$$
\left|E X_{k}^{m}\right| \leq \frac{m !}{2} \sigma_{k}^{2} H^{m-2}, \quad k=1, \ldots, n,
$$

then we have

$$
\begin{gathered}
P\left(\sum_{i=1}^{n} X_{i} \geq x\right) \leq \exp \left(-x^{2} / 4 \sum_{i=1}^{n} \sigma_{i}^{2}\right) \quad 0 \leq x \leq \sum_{i=1}^{n} \frac{\sigma_{k}^{2}}{H}, \\
P\left(\sum_{i=1}^{n} X_{i} \leq-x\right) \leq \exp \left(-x^{2} / 4 \sum_{i=1}^{n} \sigma_{i}^{2}\right) \quad 0 \leq x \leq \sum_{i=1}^{n} \frac{\sigma_{k}^{2}}{H} .
\end{gathered}
$$

Proof. Based on Theorem 2.5 in Taylor, 2002, P.643-656 and Lemma 1 in Sung, 2001, P.413-419, this Lemma is easy to prove.

Lemma 3. Suppose $\left\{X_{n}, n \geq 1\right\}$ are the exchangeable random variables and there exist $h>0, r>0$ such that

$$
E\left[\exp \left(h(x)^{r}\right)\right]<\infty .
$$

Let $\left\{X_{n i}, 1 \leq i \leq n, n \geq 1\right\}$ are the exchangeable random variables that satisfy

$$
\operatorname{Cov}\left(f_{1}\left(X_{n 1}\right), f_{2}\left(X_{n 2}\right)\right) \leq 0
$$

with $E X_{n i}=0,1 \leq i \leq n, n \geq 1$; and $\left\{a_{n i}\right\}, 1 \leq i \leq n, n \geq 1$ are real constant array that satisfy

(i). There exist $\beta$ with $0<\beta \leq r$ and $\left\{u_{n}, n \geq 1\right\}$ with $\lim _{n \rightarrow \infty} u_{n}=0$ such that

$$
\left|a_{n i} X_{n i}\right| \leq \frac{u_{n}\left|X_{i}\right|^{\beta}}{\log n}, \text { a.s. }
$$

(ii). There exists $\delta>0$ and array $\left\{v_{n}\right\}$ that satisfy $\lim _{n \rightarrow \infty} v_{n}=0$ such that

$$
X_{n i}^{2} \sum_{i=1}^{n} a_{n i}^{2} \leq \frac{v_{n}\left|X_{i}\right|^{\delta}}{\log n}, \text { a.s. }
$$

then

$$
\sum_{i=1}^{n} a_{n i} X_{n i} \rightarrow 0
$$

a.s. $n \rightarrow \infty$.

Proof. Based on Theorem 2.5 in Taylor, 2002, P.643-656 and Theorem 18 in Petrov, 1991,83-84, the lemma is easy to prove.

Lemma 3. Suppose $\left\{X_{n}, n \geq 1\right\}$ are the exchangeable random variables and there exist $h>0, r>0$ such that

$$
E\left[\exp \left(h(x)^{r}\right)\right]<\infty,
$$

$\left\{X_{n i}, 1 \leq i \leq n, n \geq 1\right\}$ are the exchangeable random variables that satisfy

$$
\operatorname{Cov}\left(f_{1}\left(X_{n 1}\right), f_{2}\left(X_{n 2}\right)\right) \leq 0
$$

with $E X_{n i}=0,1 \leq i \leq n, n \geq 1$; and $\left\{a_{n i}\right\}, 1 \leq i \leq n, n \geq 1$ are real constant array that satisfy

(1). $E\left[\exp \left(h(x)^{r}\right)\right]<\infty$.

(2). There exist $\beta$ with $0<\beta \leq r$ and constant $c>0$ such that

$$
\left|a_{n i} X_{n i}\right| \leq \frac{c\left|X_{i}\right|^{\beta}}{\log n}, \text { a.s. }
$$

(3). There exists $\delta>0$ and array $\left\{v_{n}\right\}$ that satisfy $\lim _{n \rightarrow \infty} v_{n}=0$ such that

$$
X_{n i}^{2} \sum_{i=1}^{n} a_{n i}^{2} \leq \frac{v_{n}\left|X_{i}\right|^{\delta}}{\log n}, \text { a.s. }
$$


then

$$
\sum_{i=1}^{n} a_{n i} X_{n i} \rightarrow 0
$$

a.s. $n \rightarrow \infty$.

\section{The main results and proof}

Theorem 1. Suppose $\left\{X, X_{n}, n \geq 1\right\}$ are the exchangeable random variables that satisfy

$$
\operatorname{Cov}\left(f_{1}\left(X_{1}\right), f_{2}\left(X_{2}\right)\right) \leq 0 .
$$

Suppose $f_{i}, i=1,2$ are functions satisfy the above rule and non-decrease with $X_{1}$ and $X_{2}, E X_{1}, \alpha p>1, p<2$, $l(x)>0$ is monotonous non-decrease function when $x \rightarrow+\infty,\left\{a_{n i}\right\}, 1 \leq i \leq n, n \geq 1$ are real constant array with $A_{\alpha, n}^{\alpha}=n^{-1} \sum_{i=1}^{n}\left|a_{n i}\right|^{2}$. Further, suppose

$$
A_{\alpha}=\lim _{n \rightarrow} A_{\alpha}, \quad n \leq \infty
$$

and

$$
E|X|^{\beta} \leq \infty
$$

and

$$
E X=0
$$

with $1<\alpha, \beta<\infty, 1<p<2$ and $\frac{1}{p}=\frac{1}{\alpha}+\frac{1}{\beta}$. Then we have

$$
\frac{1}{n^{1 / p}} \sum_{i=1}^{n} a_{n i} X_{i} \rightarrow \infty \text { a.s. } n \rightarrow \infty .
$$

Proof. Without loss of generality, for any $1 \leq i \leq n, n \geq 1$, suppose $a_{n i}>0$. As $\left\{X, X_{n}, n \geq 1\right\}$ are the exchangeable random variables, and $a_{n 1} X_{1}, \ldots, a_{n n} X_{n}$ also satisfy

$$
\operatorname{Cov}\left(f_{1}\left(a_{n 1} X_{1}\right), f_{2}\left(a_{n 2} X_{2}\right)\right) \leq 0
$$

and $1<\alpha, \beta<\infty, 1<p<2$, and $\frac{1}{p}=\frac{1}{\alpha}+\frac{1}{\beta}$. Then $p<\alpha \wedge \beta \wedge 2$. From Yang, 2000, P.218-223 and (1) we have

$$
E\left|n^{-1 / p} \sum_{i=1}^{n} a_{n i} X_{i}\right|^{\alpha \wedge \beta \wedge 2} \leq C n^{\alpha \wedge \beta \wedge 2 / p} \sum_{i=1}^{n}\left|a_{n i}\right|^{\alpha \wedge \beta \wedge 2} E X^{\alpha \wedge \beta \wedge 2} \leq C n^{\alpha \wedge \beta \wedge 2 / p+1} A_{\alpha \wedge \beta \wedge 2, n}^{\alpha \wedge \beta \wedge 2} \rightarrow 0, n \rightarrow \infty .
$$

Therefore, $n^{-1 / p} \sum_{i=1}^{n} a_{n i} X_{i} \rightarrow 0, n \rightarrow \infty$. From the symmetrized inequality proved in Lemma 14 in Petrov, 1991, P.83-84, we know that, in order to prove $n^{-1 / p} \sum_{i=1}^{n} a_{n i} X_{i} \rightarrow 0, n \rightarrow \infty$, we just need to prove

$$
n^{-1 / p} a_{n i} X_{i}^{S} \rightarrow 0, \quad \text { a.s. } n \rightarrow \infty,
$$

where $X_{i}^{S}$ is the symmetrized form of $X_{i}$. From Lemma 3 in Chi, 1997, P.199-203. we have the symmetrized series of

$$
\operatorname{Cov}\left(f_{1}\left(X_{1}\right), f_{2}\left(X_{2}\right)\right) \leq 0
$$

also satisfy the inequality, i.e.

$$
\operatorname{Cov}\left(f_{1}\left(X_{1}^{S}\right), f_{2}\left(X_{2}^{S}\right)\right) \leq 0 .
$$

Without loss of generality, we assume that $\left\{X_{n}, n \geq 1\right\}$ are the symmetrized exchangeable random variables that satisfy $\operatorname{Cov}\left(f_{1}\left(X_{1}\right), f_{2}\left(X_{2}\right)\right) \leq 0$ for all $1 \leq i \leq n, n \geq 1$. Letting

$$
\begin{gathered}
X_{I}^{\prime}=X_{i} I\left(\left|X_{i}\right| \leq n^{1 / \beta}\right)+n^{1 / \beta} I\left(X_{i}>\beta\right)-n^{1 / \beta} I\left(X_{i}<-n^{1 / \beta}\right), \\
X_{I}^{\prime}=X_{i} I\left(\left|X_{i}\right|>n^{1 / \beta}\right)-n^{1 / \beta} I\left(X_{i}>\beta\right)+n^{1 / \beta} I\left(X_{i}<-n^{1 / \beta}\right), \\
\bar{X}_{i}^{n}=X_{i} I\left(\left|X_{i}\right|>n^{1 / \beta}\right), \\
a_{n i}^{\prime}=a_{n i} I\left(\left|a_{n i}\right| \leq n^{1 / \alpha}\right), \\
a_{n i}^{\prime \prime}=a_{n i}-a_{n i}^{\prime}=a_{n i} I\left(\left|a_{n i}\right|>n^{1 / \alpha}\right) .
\end{gathered}
$$

Then we have

$$
\sum_{i=1}^{n} a_{n i} X_{i}=\sum_{i=1}^{n} a_{n i}^{\prime} X_{i}^{\prime}=\sum_{i=1}^{n} a_{n i}^{n} X_{i}^{\prime}+\sum_{i=1}^{n} a_{n i} X_{i}^{n}
$$


As $\frac{1}{p}=\frac{1}{\alpha}+\frac{1}{\beta}, \beta=\frac{\alpha}{\alpha-1}\left\{1+\beta\left(1-\frac{1}{p}\right)\right\},\left|\bar{X}_{i}\right|^{\prime \prime}<\left|\bar{X}_{i}\right|^{\prime \prime} \frac{\alpha \beta}{\alpha-1} n^{-\left(1-\frac{1}{p}\right)}$, and $E|X|^{\beta}<\infty$, which is equivalent to $\sum_{n=1}^{\infty} P\left(|X|^{\beta}>n\right)<\infty$.

From Borel-Cantelli Lemma, we have $P\left(|X|^{\beta}>\right.$ n, i.o. $)<\infty=0$, hence

$$
\frac{1}{n} \sum_{i=1}^{n}\left|\bar{X}_{i}^{\prime \prime}\right|^{\beta} \rightarrow \text { 0,a.s. }(n \rightarrow \infty) \text {. }
$$

From Hölder inequality, $\left|X_{i}^{\prime}\right| \leq\left|\bar{X}_{i}^{\prime \prime}\right|$ and $\left|\bar{X}_{i}^{\prime \prime}\right| \leq\left|\bar{X}_{i}^{\prime \prime}\right|^{\beta(\alpha-1) / \alpha} n^{-\left(1-\frac{1}{p}\right)}$ and

$$
\frac{1}{n} \sum_{i=1}^{n}\left|\bar{X}_{i}^{\prime \prime}\right|^{\beta} \rightarrow 0, \text { a.s. }(n \rightarrow \infty)
$$

then we have

$$
n^{-1 / p}\left|\sum_{i=1}^{n} a_{n i} \bar{X}_{i}^{\prime \prime}\right| \leq n^{-1 / p} \sum_{i=1}^{n}\left|a_{n i}\right|\left|\bar{X}_{i}^{\prime \prime}\right| \leq n^{-1} \sum_{i=1}^{n}\left|a_{n i}\right|\left|X_{i}^{\prime \prime}\right|^{\beta(\alpha-1) / \alpha} \leq A_{\alpha, n}\left(\frac{1}{n} \sum_{i=1}^{n}\left|X_{i}^{\prime \prime}\right|^{\beta}\right)^{(\alpha-1) / \alpha}, \text { a.s.n } \rightarrow \infty .
$$

Therefore, we have

$$
n^{-1 / p} \sum_{i=1}^{n} a_{n i} \bar{X}_{i}^{\prime \prime} \leq n^{-1 / p} \rightarrow 0, \text { a.s. } n \rightarrow \infty .
$$

As $1<p<2$, and $\frac{1}{p}=\frac{1}{\alpha}+\frac{1}{\beta}$, and $\alpha \vee \beta<2$, so

$$
1+\frac{(2-\alpha)^{+}}{\alpha}+\frac{(2-\beta)^{+}}{\beta}= \begin{cases}\frac{2}{\alpha \wedge \beta}, & \alpha \wedge \beta<2, \\ 1 & \alpha \wedge \beta \geq 2,\end{cases}
$$

Therefore, we have

$$
\sum_{i=1}^{n} E\left(a_{n i}^{\prime} X_{i}^{\prime}\right)^{2} \leq C_{n} A_{\alpha \wedge 2, n}^{\alpha \wedge 2} n^{\frac{(2-\alpha)^{+}}{\alpha}+\frac{(2-\beta)^{+}}{\beta}}\|X\|_{\beta \wedge 2}^{\beta \wedge 2}=O\left(\max \left\{n^{2 / \alpha}, n, n^{2 / \beta}\right\}\right) .
$$

Moreover, for any $1 \leq i \leq n, n \geq 1$, we have $\left|n^{-1 / n} a_{n i}^{\prime} X_{i}^{\prime}\right| \leq n^{1 / \alpha} n^{1 / \beta} n^{-1 / p}$, and $\max \left\{n^{2 / \alpha}, n, n^{2 / \beta}=O\left(n^{2 / p} \log ^{-2} n\right)\right.$. From Lemma 2, for sufficient small $\varepsilon$ and sufficient large $n$, we have

$$
P\left(n^{1 / p} \sum_{i=1}^{n} a_{n i}^{\prime} X_{i}^{\prime}>\varepsilon\right) \leq \exp \left(\frac{-\varepsilon^{2}}{4 n^{-2 / p} O\left(\max \left\{n^{2 / \alpha}, n, n^{2 / \beta}\right\}\right)}\right) \leq \exp \left(-\varepsilon^{2}(\log n)^{2}\right) .
$$

By the same procedures, we can also prove that

$$
P\left(n^{-1 / p} \sum_{i=1}^{n} a_{n i}^{\prime} X_{i}^{\prime}<-\varepsilon\right) \leq \exp \left(-\varepsilon^{2}(\log n)^{2}\right) .
$$

Therefore,

$$
\sum_{i=1}^{n} P\left(n^{-1 / p} \sum_{i=1}^{n} a_{n i}^{\prime} X_{i}^{\prime}>\varepsilon\right)<\infty
$$

From the above inequality, we have

$$
n^{-1 / p} \sum_{i=1}^{n} a_{n i}^{\prime} X_{i}^{\prime} \rightarrow 0, \quad \text { a.s. } n \rightarrow \infty .
$$

Also, based on $\alpha>1$ and $\frac{1}{p}=\frac{1}{\alpha}+\frac{1}{\beta}$, we have

$$
n^{-1 / p}\left|\sum_{i=1}^{n} a_{n i}^{\prime \prime} X_{i}^{\prime}\right| \leq n^{-1 / p} n^{1 / \beta} \sum_{i=1}^{n} a_{n i} I\left(a_{|n i|}>n^{1 / \alpha}\right) \leq n^{-1 / p+1 / \beta} n^{(1-a l p h a)} / \alpha \sum_{i=1}^{n}\left|a_{n i}\right|^{\alpha}=A_{\alpha, n}^{\alpha} .
$$

Then from (4),(5),(6),(7), we have

$$
\lim _{n \rightarrow \infty} \sup n^{-1 / p}\left|\sum_{i=1}^{n} a_{n i} X_{i}\right| \leq A_{\alpha}^{\alpha} \quad \text { a.s. } \quad n \rightarrow \infty
$$

By replacing $X_{i}$ with $t X_{i}$, we have

$$
\lim _{n \rightarrow \infty} \sup n^{-1 / p}\left|\sum_{i=1}^{n} a_{n i} X_{i}\right| \leq \frac{A_{\alpha}^{\alpha}}{t} \quad \text { a.s. } \quad n \rightarrow \infty .
$$


Let $t \rightarrow \infty$, we have $n^{-1 / p} \sum_{i=1}^{n} a_{n i} X_{i} \rightarrow \infty$ a.s. $n \rightarrow \infty$, i.e. the inequality (3) is true.

\section{References}

Bai, Z., \& D. Cheng, P. E. (2000). Marcinkiewicz Strong Laws for Linear Statistics. Statist Probab Lett, 46, $105-112$.

Chi, X., \& Su, C. (1997). A weak law of large number of identical distribution NA series. Applied Probab and Statist, 13, 199-203.

Fintti, B. De. (1930). Funzione Caratteristica Di Unfenomeno Aleatorio. Atti Accad. NaZ. Lincei Rend. cl. Sci. Fis. Mat. Nat. 4, 86-133.

Lin, Z. Y., L, C. R. \& Su, Z. G. (1999). The basic material for probability limit theory. Higher Education Press, Beijing, 85-86.

Petrov, B. (1991). Sums of Independent Random Variable. Hefei:USTC Press, 83-84.

Sung, S. H. (2001). Strong Laws for weighted suns of i.i.d. random variables. Statist Probab Lett, 52, 413-419.

Taylor, R. L. \& Patterson, R. F. (2002). A strong laws of large numbers for arrays of rowwise negatively dependent random variables. Stochastic Anal. Appl. 20, 643-65.

Wang, L., \& Liu, J.Q. (2001). Convergence on bivariate weighted sums of row-wise exchangeable random variables. Journal of Harbin Institute of Technology,33, 826-829.

Wu, Y. Q. (2006). The probability limit theory of composite series. Academic Press, 132-133.

Yang, S. Z. (2000). The matrix inequality of partial sum of random variables. Science in China, 30, 218-223. 